Supporting information

\title{
Facile production of hexagonal boron nitride nanoparticles by cryogenic exfoliation
}

Ngoc My Hanh Duong, ${ }^{1}$ Evgenii Glushkov, ${ }^{2}$ Andrey Chernev, ${ }^{2}$ Vytautas Navikas, ${ }^{2}$ Jean

Comtet, ${ }^{2}$ Minh Anh Phan Nguyen, ${ }^{1}$ Milos Toth ${ }^{1}$, Aleksandra Radenovic, ${ }^{2}$ Toan Trong Tran, ${ }^{1 *}$ and Igor Aharonovich ${ }^{1, *}$

1School of Mathematical and Physical Sciences, University of Technology Sydney, Ultimo, NSW, 2007, Australia

${ }^{2}$ Laboratory of Nanoscale Biology, Institute of Bioengineering, School of Engineering, École Polytechnique Fédérale de Lausanne (EPFL), 1015 Lausanne, Switzerland

*Corresponding author: igor.aharonovch@uts.edu.au or TrongToan.Tran@uts.edu.au

\section{TEM sample characterization}

a

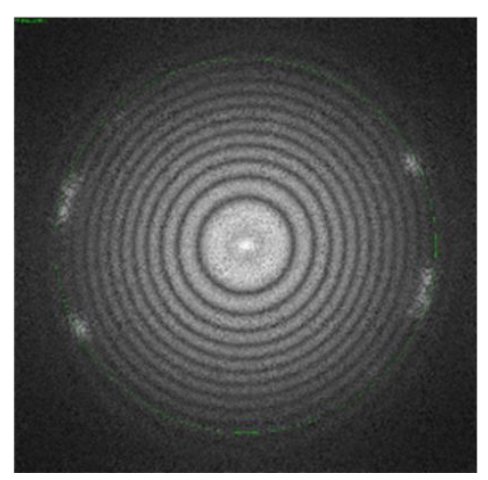

b

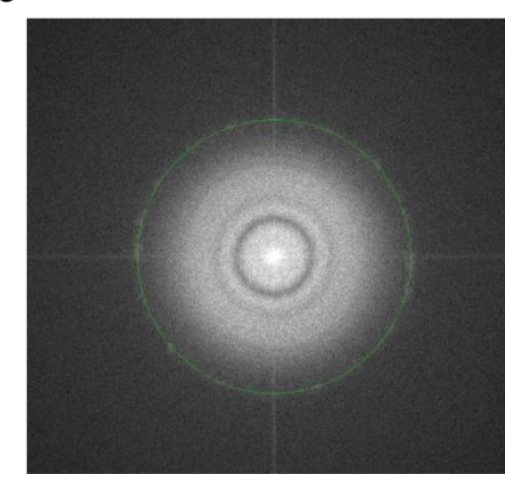

C

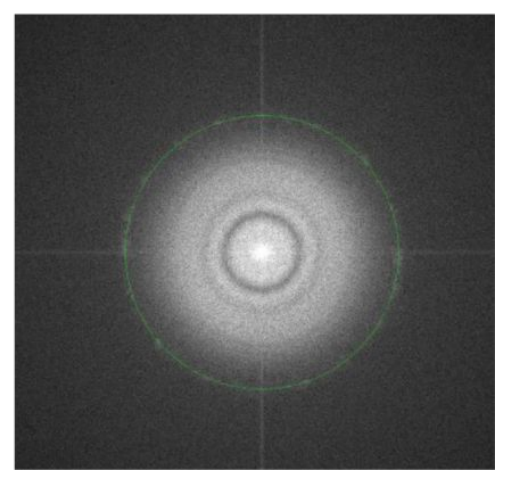

Figure S1. FFT of the HRTEM images shown on Fig. 1 (samples S1, S2 and S3) with dedicated maxima, corresponding to the lattice parameters of $\mathrm{h}-\mathrm{BN}(2.54 \mathrm{~A})$. 
Spectral characteristics of quantum emitters in hBN nanoparticles
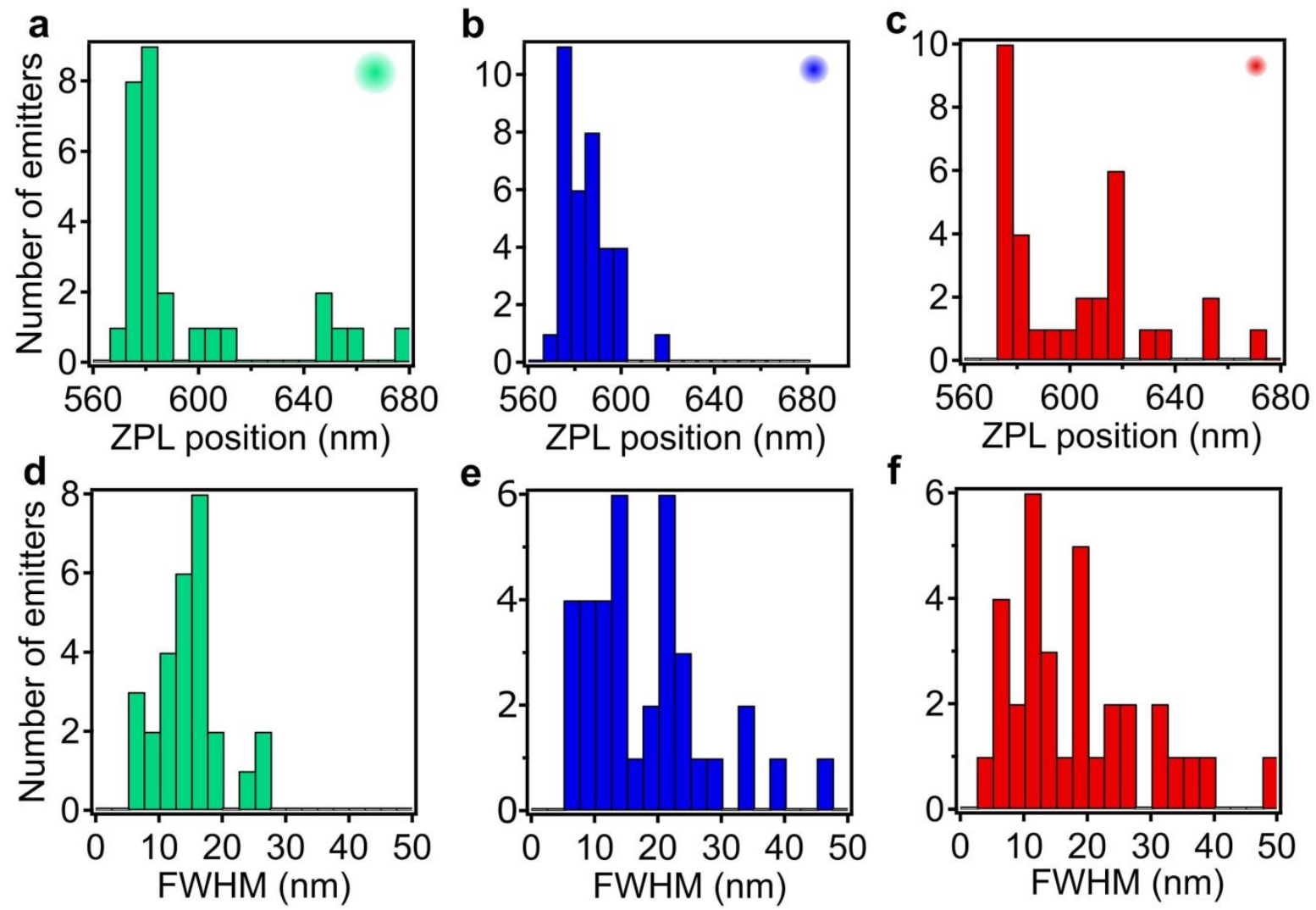

Figure S2. Summary of spectral characteristics of quantum emitters in hBN nanoparticles. (a - c) Histograms of zero-phonon line position obtained from the spectra of emitters in sample S1, S2 and S3, respectively. ( $-\mathrm{f}$ ) Histograms of full-width-at-half-maximum extracted from the spectra of emitters in sample S1, S2, and S3, respectively. All the measurements were taken only with a 568-nm long-pass filter in the collection path at room-temperature. 


\section{Dynamic Light Scattering Measurements}
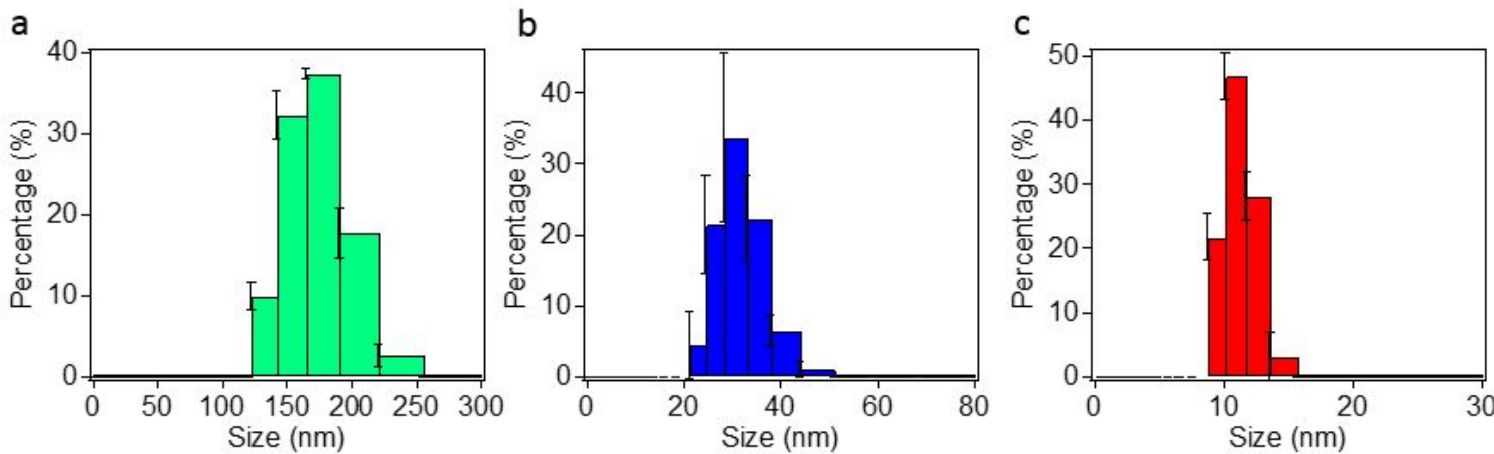

Figure S3. Size distribution from DLS measurements of different hBN samples a) S1, b) S2, c) S3. Error bars represent standard deviations obtained from three measurements of the same sample. The average sizes of the samples are $159 \mathrm{~nm}, 25 \mathrm{~nm}$, and $9 \mathrm{~nm}$ respectively. The sizes of nanoparticles measured in DLS are larger than the size measured by AFM because the size distribution seen in DLS is the hydrodynamic size of the particles so any aggregation will result in a larger size. 